combination prevention and across the full prevention cascade, and that without that focus, sub-optimal decisions may be taken.

\section{SO1.5 THE ARCHITECTURE FOR PREVENTION AND THE ROLE OF TARGETS AND INDICATORS}

Mitchell Warren*. AVAC Global Advocacy for HIV Prevention, New York, USA

\subsection{6/sextrans-2015-052270.21}

There are effective means to prevent every mode of transmission; political commitment on HIV continues to be strong; and financing for HIV programs in low- and middle-income countries increased has surpassed US\$17 billion. However, amidst recurring calls for a comprehensive, integrated and sustained AIDS response, funding, targets and delivery are focusing disproportionately on treatment. This presentation will describe the financing, leadership and implementation/delivery architecture for HIV prevention as it exists today, and as it needs to be developed in order to fully realise the potential of existing, emerging and on-the-horizon HIV prevention options.

Beginning with an exploration of the current architecture of HIV prevention financing, the presentation will highlighting current gaps, such as the recent scale back of PEPFAR funding for VMMC and shortfalls in funding and planning for PrEP product introduction, citing these examples to highlight broader issues in the architecture as it exists (e.g. the gap between positive research results and substantive, strategic planning to move to implementation), individual interventions (e.g. VMMC) being "owned" by single donors, and then in jeopardy when funding fluctuates. The presentation will also explore the leadership, describing the actors that influence policy, programming and messaging at global, regional and national levels.

Finally, this presentation will examine the gaps and strengths in current implementation and delivery architecture, focusing on what is available and/or needed to deliver prevention and on the targets that need to be set to help drive implementation and financing; how targets need to be tailored to each specific intervention; and highlight the potential for conceiving and building a comprehensive prevention delivery platform that maximises the use of existing interventions and supports the rapid and effective integration of new options as they become available.

\section{S02 - Education about sex and relationships: new directions in school and beyond}

\section{SO2.1 SEX AND RELATIONSHIPS EDUCATION IN SCHOOLS: A KEY COMPONENT OF SEXUAL HEALTH PROMOTION}

Peter Aggleton*. UNSW Australia, Sydney, Australia

10.1136/sextrans-2015-052270.22

Despite scientific evidence of effectiveness, there continues to exist controversy about sex and relationships education (SRE). Much of this relates to misunderstandings about the form that good quality SRE takes and what it aims to achieve. The evidence reveals that well designed and effectively implemented programs of comprehensive sexuality education have the potential to bring about beneficial outcomes for young people. But such programs often work best when they are implemented through partnership between education and health. Building on the framework offered by WHO in its guidance on Developing Sexual Health Programmes, this presentation highlights some of the ways in which this can be achieved: through the provision of in-school clinics and other health services; through effective signposting and fast-track routes to relevant services; and through health professionals' involvement in the life of the school. It signals the importance of understanding differences in culture and tradition between the education and health sectors, stressing how teachers, educational administrators and health professionals often understand 'intervention' and 'education' quite differently. Finally, it will stress the importance of respect for difference, and respect for the 'other' in work in schools.

\section{S02.2 RELIGIOUS AND FAITH-BASED BELIEFS: A HELP OR A HINDRANCE IN SEXUAL HEALTH EDUCATION?}

Mary Lou Rasmussen*. Monash University, Melbourne, Australia

\subsection{6/sextrans-2015-052270.23}

The Reverend Debra Haffner, a former CEO of SIECUS, and the Director of the Religious Institute on Sexual Morality, Justice and Healing objects to the The National Sexuality Education Standards for US Public Schools (2012) published by The Guttmacher Institute in the United States. Haffner questions these standards because they fail to account for values and religious influences on sexuality education and religion. The absence of explicit references to religion and values in such standards reflect longstanding debates about the place of religion within secular states, and about the privatisation of religion and belief. These debates also impact perceptions about the role of religion in sexuality education and shape debates about the place of reason and science in sexuality education.

Haffner's theological commitment to comprehensive sexuality education is accompanied by an expressed desire for a valuesbased framework for sexuality education. Unlike Haffner, I have no theological commitments in relation to sexuality education provision. However, I have come to question progressive sexuality education standards that do not explicitly engage with questions of faith, belief and their relationship to values. What are the grounds for this separation? Are there ways in which religion and values can be usefully incorporated in progressive sexuality education? Should such topics be left behind in the production of a progressive sexuality education?

\section{S02.3 RESPECTING GENDER AND SEXUAL DIFFERENCE TO PROMOTE SEXUAL HEALTH: MAKING SCHOOLS SAFE SPACES FOR ALL}

Kerry Robinson*. University of Western Sydney, Sydney, Australia

10.1136/sextrans-2015-052270.24

Drawing on international evidence of best practice, and recent and ongoing research funded by the Australian Research Council (ARC) and the Young and Well Collaborative Research Centre (CRC), this presentation highlights how teachers and health professionals can most effectively engage with, and approach gender, sexuality, and sexuality education, to ensure that schools offer relevant and informative education, and safe and supportive environments for all pupils irrespective of gender and sexual identity. 